\title{
VARIACIÓN LINGÜÍSTICA Y TRADUCCIÓN. ANÁLISIS DEL \\ MODELO PUBLICITARIO DE RED BULL: GIGI E LA MAMMA \\ Beatriz Moroño Prieto \\ Universidad de Málaga
}

\begin{abstract}
The present article aims to be a study on the translation of language variation in an advertisement medium. It intends to analyze its translation into different languages taking the Italian language as the basis and the Spanish, French and English versions as translations, and observing what solutions have been adopted in order to achieve the transfer of the language variation.
\end{abstract}

KEYWORDS: translation, language variation, advertising text.

\section{RESUMEN}

El presente artículo pretende ser un estudio sobre la traducción de la variación lingüística en un medio publicitario, y analizar la traducción del mismo en varios idiomas; tomando como base el anuncio en lengua italiana y como traducciones los anuncios en español, francés e inglés, y observar cuáles son las soluciones tomadas para conseguir el trasvase de la variación lingüística.

PALABRAS CLAVES: traducción, texto publicitario, variación lingüística

\section{INTRODUCCIÓN AL ESTUDIO}

Antes de abordar directamente la introducción del presente artículo, nos gustaría proponer una serie de preguntas, las mismas que nos hemos planteado para la realización del estudio de este artículo y que han sido parte de las motivaciones que nos han llevado a redactarlo: ¿Por qué estudiar la traducción de la variación lingüística en los medios audiovisuales? ¿Por qué hemos elegido este anuncio publicitario? ¿Se logra el trasvase de la variación lingüística en este medio audiovisual? ¿Qué posibles soluciones se dan para la traducción del dialecto? Esperamos dar una humilde respuesta a través de las conclusiones del estudio de este artículo, que quiere condensar en pocas 
palabras lo que se pretende analizar y el alcance de la investigación llevada a cabo.

Este artículo pretende abordar la problemática de la traducción de la variación lingüística. La búsqueda de rasgos lingüísticos supuestamente equiparables en la lengua meta que traten de reflejar la información que contiene la variación del texto origen es fuente de discusiones no sólo sobre la viabilidad de esta opción, sino sobre todo sobre su posterior impacto sobre el sistema meta. Por otro lado, la desaparición de rasgos marcados, tampoco se ven claramente favorecidas, ya que muchos autores desaprueban la pérdida de información lingüística y socio-cultural en el proceso de trasvase.

En este artículo tratamos de ofrecer una síntesis de las aproximaciones más relevantes que se han dado en los estudios de traducción a la variación. Dado que los estudios de traducción en sus inicios tomaron gran parte de sus postulados teóricos de investigaciones lingüísticas de carácter más formal, mencionamos éstas por su relevancia.

Vamos a ofrecer una exposición de los postulados teóricos de los estudios de cada una de las variaciones (diacrónica, diatópica, diastrática, diamésica y diafásica) y de los estudios de la traducción de la variación lingüística.

En este trabajo, vamos a partir de un enfoque traductológico descriptivo y realizaremos un recorrido por algunos de los problemas que plantean la traducción de cada una de las variaciones en un modelo publicitario (Análisis del modelo publicitario de Red Bull «Gigi e la mamma» en italiano) y veremos cuáles han sido las soluciones escogidas para cada caso traducido (el mismo modelo publicitario en español, en francés y en inglés).

Este artículo pretende realizar un acercamiento interdisciplinar entre la lingüística, la traducción y los medios audiovisuales y más concretamente los medios audiovisuales publicitarios.

\section{2. ¿QUÉ ES EL TEXTO?}

Entendemos el texto como unidad de carácter lingüístico emitida intencionadamente por un hablante en una situación concreta y con una finalidad determinada. Siendo la unidad lingüística más amplia y que engloba a todas las demás.

\section{1. ¿CUÁLES SON LAS PROPIEDADES DEL TEXTO?}

En este punto, vamos a hacer un estudio de las propiedades del texto: la adecuación, cuya característica principal es que los textos estén bien construidos desde el punto de vista comunicativo; la coherencia, siendo la 
propiedad que hace que el texto pueda ser percibido como una unidad comunicativa y no como una sucesión de enunciados inconexos y la cohesión, siendo conexión que, mediante recursos lingüísticos, se establece entre las partes de un texto, de manera que sean entendidas por el receptor como integrantes de una totalidad y no como independientes y desconectadas entre sí.

Dentro de la adecuación, es dónde haremos el estudio de las funciones del lenguaje (función referencial, expresiva, apelativa, fática, metalingüística y estética) y de las variaciones lingüísticas: La variación diacrónica, determinada por la evolución de la lengua en el transcurso del tiempo así como por los cambios que se producen dentro de una generación. Se producen a este respecto cambios fonéticos, ortográficos, sintácticos así como cambios de significado. La variación diatópica, hace referencia a las diferentes maneras de usar la lengua que tienen los hablantes de distintas zonas geográficas. Diferentes sub-sistemas que se refieren a los distintos usos y a las diferentes comunidades que hablan un mismo idioma (diferentes maneras de adaptarse a las circunstancias en las que se encuentran). Depende mucho de la procedencia geográfica del individuo, refiriéndose a las variedades dialectales. La variación diastrática, relacionada con la situación social, económica así como la educación. También denominada sociolecto, o dialectos sociales. La variación diamésica, estudia la observación de las variedades de las lenguas en función del medio utilizado para la comunicación, es decir, la tradicional distinción entre medio escrito (gráfico) y medio hablado (oral). La variación diafásica, se produce cuando el hablante adapta su expresión a la situación comunicativa. Determina los usos que de la lengua hacen los hablantes (y no los usuarios): es el hablante nativo de una lengua quien mejor sabrá usar la lengua según la situación, eligiendo según el caso un determinado repertorio lingüístico.

\section{EL TEXTO PUBLICITARIO}

La traducción de espots publicitarios es un género más de traducción audiovisual, dónde la combinación de imagen y sonido depende de un fin único claramente persuasivo. El término spot publicitario es lo que se designa a cada película corta emitida en los cortes entre programas televisivos, y su finalidad es proporcionar al producto o a un servicio su promoción.

La característica principal de los espots publicitarios es que tienen dos códigos, uno visual, y otro oral, ambos combinados y subordinados al soporte para el cual son diseñados, así como la principal función que define al texto publicitario es la persuasiva.

El código visual del espot se manifiesta de maneras diversas: la imagen móvil, el código cromático, las imágenes fijas, el código tipográfico, etc. 
El código oral del espot publicitario se caracteriza por su complejidad, se presenta de forma sonora mediante la voz en off, mediante el texto de una canción, etc.

La relación entre los aspectos visuales y orales, y su significado global, marcan las diferentes pautas que hay que tener en cuenta a la hora de traducir, siendo la mayor dificultad a la que se enfrenta el traductor ya sea de un espot publicitario u otro texto de carácter audiovisual, la de mantener en todos los componentes del mensaje un sincronismo de significado (Mayoral, Kelly y Gallardo, 1986).

Por tanto, la traducción de espots publicitarios ha de tener en cuenta todas las operaciones tanto en el plano verbal como no verbal.

\section{LA TRADUCCIÓN DE LA VARIACIÓN LINGÜÍSTICA}

Tanto la teoría de la traducción como la lingüística teórica o la sociolingüística estudian aspectos relacionados con la lengua, las disciplinas lingüísticas tienen como objeto la descripción de una lengua y la traductología tiene como objeto el estudio del proceso de la traducción, que es un proceso comunicativo entre lenguas $\mathrm{y} / \mathrm{o}$ culturas diferentes bajo un encargo profesional.

Hablar hoy de variación lingüística exige, que nos detengamos a explicar qué es lo que entendemos por ella, pues esta disciplina nacida de la unión de diversas disciplinas, mantuvo, por su irregularidad y su carácter externo, malas creencias por parte de los lingüistas, que no poniendo en duda de su existencia, llegaron a considerarla de manera no adecuada al explicar el funcionamiento de las lenguas.

Pocos han sido los que se han ocupado de definir la variación lingüística, aunque sí han sido muchos los que la han estudiado, han trabajado con ella y la han descrito.

A continuación ofreceremos una breve pero exhaustiva bibliografía de los principales autores y textos que pueden ser como referente para el presente trabajo, partiremos de las aportaciones a los estudios de la variación lingüística desde los estudios sociolingüisticos de la variación, y destacaremos autores como Labov y su obra pionera The social Stratification of English in New Cork City (1966). Halliday, McIntoch y Strevens estudian la variación lingüística en su libro The linguistic Sciences and Language Teaching (1964: 75-110). Michael Gregory y Susan Carroll, en su obra Language and Situation: Language Varieties and Their Social Contexts (1978) y otros como Hatim y Mason (1990).

Señalaremos las aportaciones a los estudios de la variación lingüística desde los estudios lingüísticos de la variación, y destacaremos autores como Georges 
Mounin, en su trabajo Les problèmes théoriques de la traduction (1963), a Eugenio Coseriu en su libro Lezioni di linguistica generale (1981).

Y para los estudios traductológicos de la variación, señalaremos autores como Eugene Nida en sus obras Languages and Dialects into which Translations Should Be Made (1947) y Reestructuring (1982), J.C. Catford, en su obra A Linguistic Theory of Translation (1965), Rosa Rabadán en su obra Equivalencia y Traducción: Problemática de la equivalencia translémica inglés-español (1991), Basil Hatim e Ian Mason, en su obra Discourse and Translator (1990) y Roberto Mayoral, en sus trabajos relacionados con la variación lingüística como Comentario a la traducción de algunas variedades de lengua (1990), o Problemas de traducción de los sistemas de referencia de segunda y tercera persona (1989) y El texto como unidad en la traducción del tabú lingüístico (1987).

\section{TRASCRIPCIÓN EN ITALIANO DEL SPOT GIGI E LA MAMMA}

La forma que vamos a utilizar para presentar la trascripción de cada modelo publicitario es utilizando el modelo que el profesor Frederic Chaume que en su libro Cine y Traducción (2004) muestra como formato para realizar y entregar las traducciones de los guiones.

Para el doblaje, la unidad de traducción que utilizamos, es la toma o take, anglicismo utilizado en el mundo profesional. Una toma suele consistir en un fragmento escrito de unos cinco a diez renglones, siguiendo una serie de convenciones, Chaume (2004):

- Un mismo actor de doblaje no puede intervenir en más de cinco renglones en la misma toma. La suma total de sus intervenciones en una misma toma no puede superar los cinco renglones tanto si son seguidos como alternos.

- La cantidad máxima de líneas de un take no puede exceder los diez renglones, siempre y cuando no haya un actor con más de cinco renglones.

- Cuando exista una pausa en el texto audiovisual que exceda los 15 segundos, el traductor o adaptador cortarán la toma y comenzarán otra nueva.

- Si existe un cambio de escena significativo, es aconsejable el cambio de toma.

- Si una toma dura más de 30 segundos, el traductor cortará la toma en dos. 
TAKE 1.

MAMMA Ue! Gigí!, ma tu si pazzo! Non andà così veloce.

GIGI Si...

MAMMA A cintura, te l'hai messa la cintura...

GIGI Si, siii.

MAMMA A benzina, come stiamo con la benzina?

GIGI C’è né mamma!

TAKE 2.

MAMMA Va chiano... Frena! Frena! State accourtu, accussì decolliamo, bellemammà!

GIGI E dai mamma! Vado a quaranta all'ora...

MAMMA Ma che quaranta l'ora! Stiamo volando. Ah! San Gennaro, e mo' bevi pure

mentre guidi, eh?

GIGI Si, mamma, bevo Red Bull, che stimola corpo e mente e ti mette le aaaaaali!

Ciao mamma!

TAKE 3.

MAMMA Gigí! A do te ne vai vulando accussi!! Chiama quando arrivi figlio mio! Va

piano, nun fa tard, m'arraccumandu, pensa a mamma toia che ti voglie tanto beeeene!

\subsection{ANÁLISIS DE LA VARIACIÓN LINGÜÍSTICA DEL TEXTO EN ITALIANO}

Vamos a realizar el análisis de las distintas variaciones lingüísticas del modelo publicitario italiano, con ejemplos extraídos del texto trascrito anteriormente.

La variación diacrónica:

Se refiere a la evolución de la lengua a lo largo del tiempo. Debido a las características de este anuncio, no hemos encontrado ninguna marca de variación diacrónica. No existen referencias a un italiano antiguo o arcaísmos.

La variación diatópica: 
Hace referencia a las diferentes maneras de emplear la lengua en uso a los hablantes de distintas zonas geográficas.

Esta variación es la que más caracteriza nuestro anuncio en italiano, el cual juega totalmente con la combinación del italiano estándar y el dialecto napolitano. Esta característica, la de mezclar ambos códigos lingüísticos, es un recurso que se utiliza en Italia para jugar con los personajes e implica muchas connotaciones. Por ejemplo: el uso del dialecto (napolitano en este caso), se utiliza para añadir humor a lo que estamos presentando, en este caso un anuncio de una bebida, pero en otros casos, también se ayudan del juego de cambio de registro, ya que el napolitano es uno de los dialectos italianos más «peculiares» y muy utilizado por famosos humoristas italianos de origen napolitano (entre todos destacamos Totó o los hermanos De Filippo). Por lo tanto, es un recurso digamos casi obligado si el texto quiere ser humorístico.

El napolitano, no es un dialecto que todas las personas en Italia comprenden. Su uso tampoco es un impedimento, ya que como de todas maneras, se ha extendido bastante, gracias a estos grandes personajes del panorama cómico en Italia, que han conseguido, que sí hay muchas expresiones que se han extendido. Pero si de todas formas no se comprende, ya sólo con el tono que se emplea, será un buen aliado para hacer más cómica una situación.

En nuestro ejemplo, en el último párrafo dónde aparece la mamma, esta figura de la madre italiana, napolitana, que se preocupa a desmesura por su hijo, caracterizada por sus gestos, moviendo brazos y gritando al cielo, la situación es muy cómica, y a la vez muy exagerada, y si a todo esto añadimos todo lo que dice la mujer en dialecto napolitano, cumple con una nota muy alta su objetivo:

MAMMA Gigí! A do te ne vai vulando accussì! Chiapa quando arrivi figlio mio! Va piano, nun fa tard, m'arraccumandu, pensa a mamma toia che ti voglie tanto beeeene!

El lenguaje que están utilizando es un lenguaje familiar:

MAMMA Ue! Gigí!, ma tu si pazzo! Non andà così veloce.

GIGI C'è né mamma!

Utilizar dialecto para comunicar en el seno de una familia es algo muy normal, que en Italia, país característico por su gran variedad de dialectos, estos dialectos se utilizan como el lenguaje familiar, utilizado de padres a hijos o entre amigos. Cabe destacar que el dialecto, se transmite de generación en generación de padres a hijos.

Queremos destacar aquí una serie de palabras que se emplean en el anuncio, en dialecto napolitano, y que vamos a traducir al italiano estándar, 
para hacer posteriormente el análisis de estas mismas palabras para comprobar cómo se han traducido, y ver si han mantenido algún rasgo cultural y lingüístico, o si simplemente se han traducido sin más, perdiendo cualquier intención de mantener la variación diatópica:

\begin{tabular}{|l|l|}
\hline $\begin{array}{l}\text { Dialecto } \\
\text { napolitano }\end{array}$ & Italiano estándar \\
\hline Si & Sei \\
\hline $\begin{array}{l}\text { Andà } \\
\text { A }\end{array}$ & $\begin{array}{l}\text { andare } \\
\text { la }\end{array}$ \\
\hline e l'hai & $\begin{array}{l}\text { te la sei (a menudo, en dialecto se falla con el uso del } \\
\text { verbo auxiliar) }\end{array}$ \\
\hline Va chiano & vai piano \\
\hline State accuortu & stai attento \\
\hline Accussì & Così \\
\hline Bellemammà & bello di mamma (contraído en italiano) \\
\hline mo' & Adesso \\
\hline Vulando & Volando \\
\hline m'arraccumandu & mi raccomando \\
\hline nun fa tard & non fare tardi \\
\hline Toia & Tua \\
\hline Voglie & Vuole \\
\hline
\end{tabular}

La variación diastrática:

Se produce por la pertenencia del hablante a un determinado grupo social que genera una fraseología y unas maneras expresivas propias.

Este tipo de variación lingüística se refleja de la misma manera que los anteriores.

Con el juego de cambio de registro, de italiano estándar a dialecto, vemos que hay una pertenencia a un grupo social por parte de la madre muy diferente al grupo social al que pertenece Gigi.

La madre que aparenta tener una edad aproximada de unos 45 años, reúne una serie de características que le hacen pertenecer a un grupo determinado y muy característico: mujer, madre, napolitana, nivel cultural medio bajo. 
Gigi, por lo contrario, no utiliza el dialecto napolitano, y se dirige siempre a su madre en italiano estándar, este personaje, reúne también una serie de características, que también le hacen pertenecer a un grupo determinado: joven, italiano que comprende el dialecto familiar napolitano, pero que no lo utiliza para hablar con su madre, posiblemente con nivel cultural más alto que el de su madre.

En este caso, se trata de una campaña publicitaria, y debemos destacar que, aparte de lo anteriormente comentado, sigue siendo un producto que hay que vender, y si nos fijamos, la única frase que Gigi dice en italiano estándar, es la frase del eslogan que caracteriza a todas las campañas de este refresco para esta marca:

GIGI Si, mamma, bevo Red Bull, che stimola corpo e mente e ti mette le aaaaaali! Ciao mamma!

La madre hace una alusión que hace que pensemos que es una verdadera y devota napolitana, al referirse al santo napolitano por excelencia, y que hace que se haga la diferencia entre un verdadero napolitano del que no lo es: San Gennaro

MAMMA Ma che quaranta l'ora! Stiamo volando. Ah! San Gennaro, e mo' bevi pure mentre guidi, eh?

La madre habla durante todo el anuncio en dialecto napolitano (en el ejemplo, hemos intentado una trascripción verídica del texto oral), y el hijo, en italiano estándar; se debe también a la diferencia de edad que tienen los dos protagonistas.

Es característico en Italia, y más en regiones donde el uso del dialecto es más difundido, que sean las personas de una cierta edad, las que se emplean en dialecto mayoritariamente, y sean los hijos, y las personas más jóvenes, los que consigan tener un bilingüismo entre el dialecto y el italiano estándar.

Junto a la edad, la variación diastrática se determina principalmente en función del grupo social y del nivel cultural de los hablantes. Los que proceden de una clase social alta, con un nivel de instrucción elevado, dominan desde niños una variación de lengua muy próxima al estándar; por el contrario, los hablantes que pertenecen a niveles más bajos de la escala social, con un nivel de instrucción escolar bajo, están mayoritariamente expuestos, durante la infancia, al uso del dialecto o a variedades del italiano distantes de la lengua estándar. Por consiguiente, mientras los hablantes de las clases altas aprenden y contribuyen a la difusión y al uso de una lengua estándar, los de las clases menos favorecidas alejan su manera de hablar de la forma estándar y usan una 
lengua socialmente más marcada, lo que los lingüistas llaman italiano popular (Caprara, 2007)

Este anuncio refleja claramente esta situación de uso del dialecto y de la lengua estándar. Vamos a ver ejemplos tomados del propio texto:

Ejemplos de la madre hablando en dialecto napolitano:

MAMMA Non andà così veloce, en vez de decir: andare così veloce

MAMMA Va chiano, en vez de decir: va piano

MAMMA Gigí! A do te ne vai vulando accussì!, en vez de decir: a dove vai...

Ejemplos de Gigi hablando en dialecto y en italiano estándar:

GIGI Vado a quaranta all'ora..., en vez de decir: andiamo a quaranta ...

GIGI Si, mamma, bevo Red Bull, che stimola corpo e mente e ti mette le aaaaaali! Ciao mamma!

La variación diamésica:

La dimensión diamésica, estudia la observación de las variedades de las lenguas en función del medio utilizado para la comunicación, es decir, la tradicional distinción entre medio escrito (gráfico) y medio hablado (oral). En este caso, la variación diamésica está presente a lo largo de todo el texto.

Cabe aclarar que siendo un anuncio, el diálogo es muy fluido, y rápido, para poder realizar el estudio de la variación lingüística, hemos tenido que trascribirlo, lo que hace que toda la riqueza oral se pierda y los acentos no se aprecien, como en el texto oral.

Pero al mismo tiempo, que sea un texto con una finalidad oral, hace que esta variación sea muy característica, ya que todo el texto en sí es una variación diamésica, teniendo como una de las características importantes del texto oral, la fluidez.

Otra de las características del texto oral en italiano, es que no se pronuncian las palabras exactamente como deberían de escribirse. Así se agiliza el lenguaje.

El dialecto napolitano tiene literatura escrita, y por lo tanto se puede escribir y transcribir, pero al uso, al ser una lengua que se trasmite oralmente, por regla general, los hablantes del napolitano, no saben cómo escribirlo. 


\section{La variación diafásica:}

Dicha también funcional, se produce cuando el hablante adapta su expresión a la situación comunicativa.

Determina los usos que de la lengua hacen los hablantes (y no los usuarios): es el hablante nativo de una lengua quien mejor sabrá usar la lengua según la situación, eligiendo según el caso un determinado repertorio lingüístico. El mismo hablante utilizará registros diferentes en una conversación con amigos en un bar o si interviene en un juicio como testigo.

En nuestro caso, el tema o campo es de ámbito cotidiano, sin ninguna especialización, con un vocabulario muy normal.

El tono es completamente familiar, tenemos como ejemplo la forma de dirigirse entre ellos, la madre utiliza un diminutivo del nombre real en italiano del joven: utiliza Gigi en vez de Luigi; y el joven llama a su madre: mamma. Ambos tienen una relación de proximidad entre ellos, al ser una conversación entre una madre y su hijo no es nada formal y tampoco existe una jerarquía entre ellos.

El modo, en este anuncio, es oral espontáneo y visual, a través de dibujos animados, aunque partimos siempre de la base que es un anuncio, que ha sido inventado por un grupo de publicistas y aunque haya sido muy estudiado, lo que el texto intenta reflejar es espontaneidad y naturalizad.

Queremos indicar que existe un lenguaje no verbal en el anuncio, un lenguaje de gestos que nos dan pistas de la procedencia de los personajes; adjuntamos los vídeos en un CD para que se puedan ver y analizar los gestos.

Destacamos el gesto de movimiento de brazos que realiza la madre cuando Gigi sale volando por la ventana, un gesto muy ligado a los gestos de los italianos en general, y mucho más característico de los italianos del sur, como en este caso, de la zona de Nápoles. Podemos decir, que el italiano, en general utiliza los gestos y los movimientos de brazos como un lenguaje que ensalza el propio lenguaje y que por sí sólo tiene su propio significado. Para nuestro estudio hemos pasado del oral al escrito para poder analizarlo.

Atendiendo a estos tres factores, podemos decir que el registro prototípico empleado a lo largo del anuncio es el coloquial.

\subsection{TRASCRIPCIÓN EN ESPAÑOL DEL SPOT GIGI E LA MAMMA}

TAKE 1.

$\begin{array}{ll}\text { MAMA } & \text { ¡Luigi no corras tanto! } \\ \text { GIGI } & \text { Vale... } \\ \text { MAMA } & ¿ \text { Te has puesto el cinturón? }\end{array}$




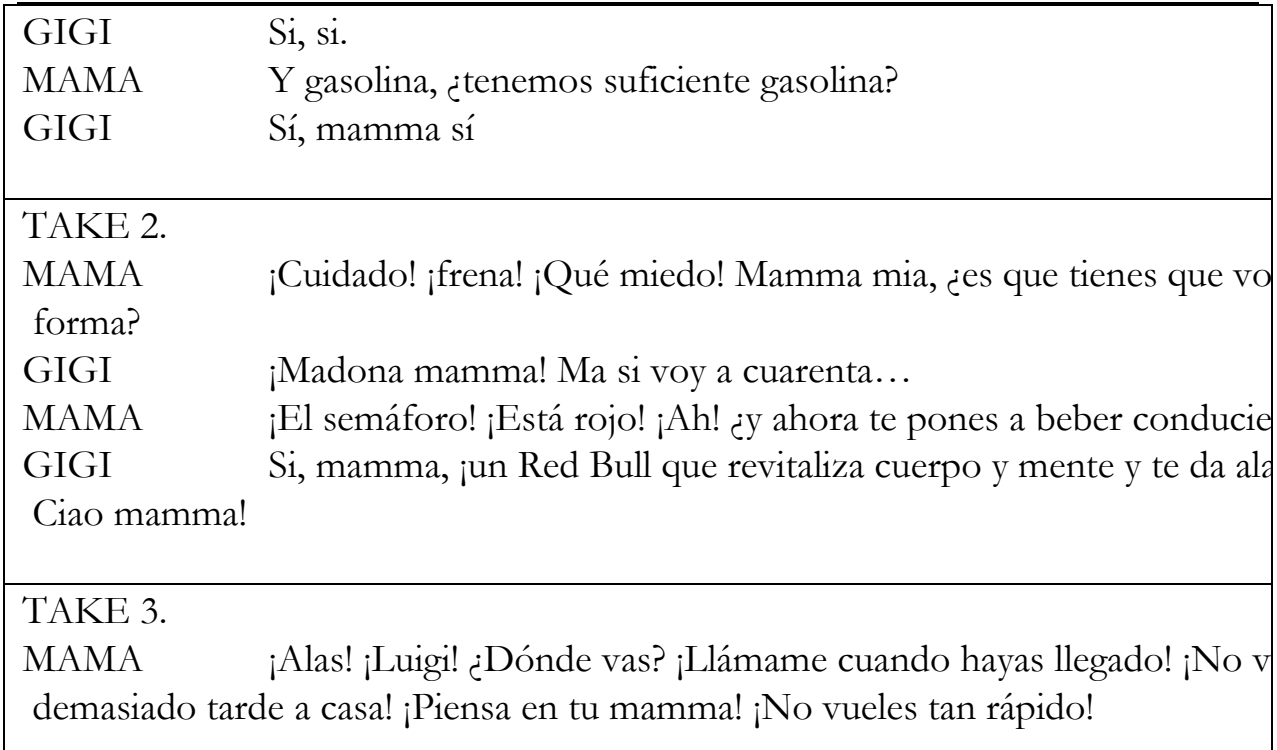

\subsection{TRASCRIPCIÓN EN FRANCÉS DEL SPOT GIGI E LA MAMMA}

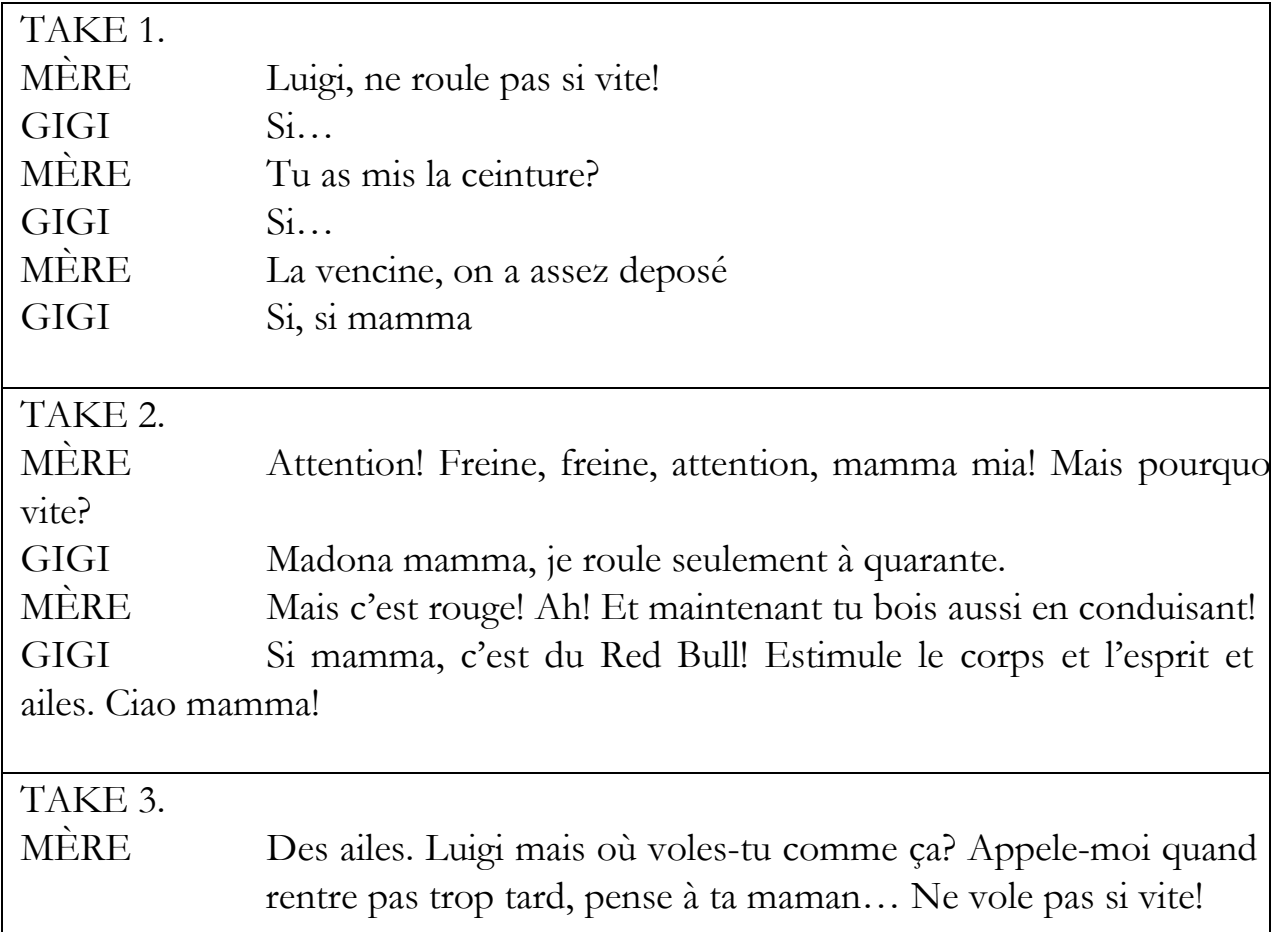




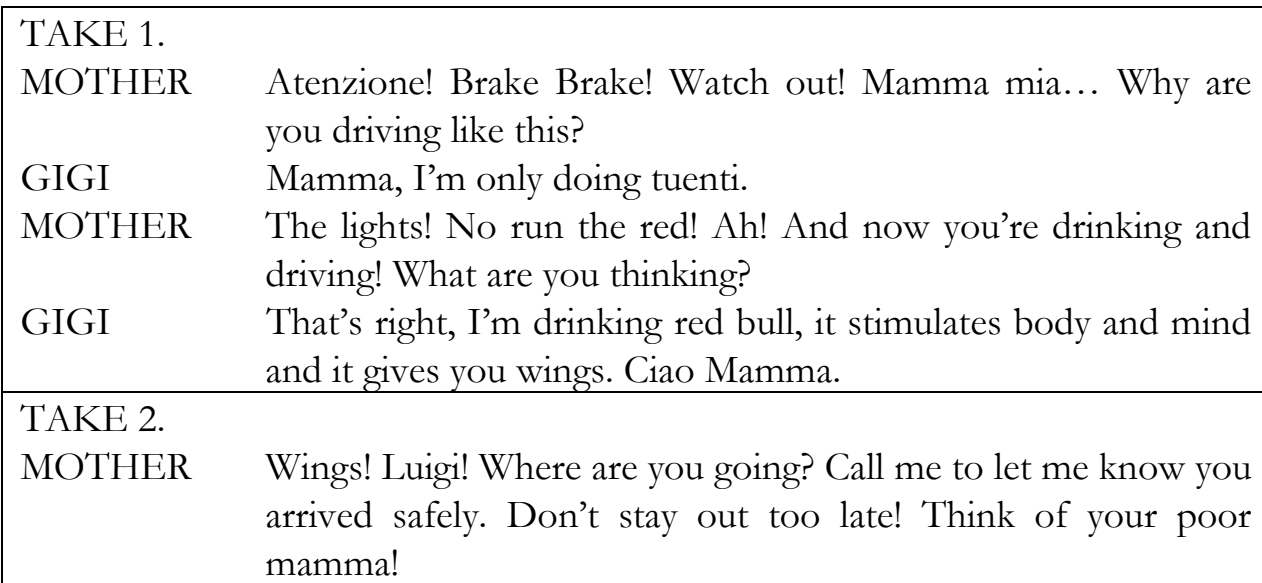

\section{ANÁLISIS COMPARATIVO DE LA VARIACIÓN LINGÜÍSTICA ENTRE LOS ANUNCIOS EN ESPAÑOL, FRANCÉS E INGLÉS}

En esta segunda parte del corpus, vamos a hacer un análisis comparativo, partiendo del estudio previo que hemos hecho del anuncio italiano, vamos a comparar los resultados obtenidos del análisis de la variación lingüística con los análisis que vamos a obtener de la traducción de la variación lingüística de los anuncios en español, en francés y en inglés.

Como primera característica, quisiéramos comentar la forma de cada anuncio: para la trascripción hemos dividido todos los anuncios en un mismo formato (Chaume, 2004) separado por takes y en los casos del italiano, español y francés, obtenemos 3 respectivamente, pero para el anuncio en inglés, sólo hay dos. Esto se debe al número permitido de renglones por cada take, como máximo, se permiten diez, si no es la misma persona que habla, que entonces serían cinco, por lo que obtenemos como resultado, que para la traducción del inglés, han modificado el guión.

Por lo tanto, ver que el ejemplo de la trascripción del anuncio en inglés, nos indica ya una primera diferencia entre la totalidad de los anuncios: no sólo se ha hecho la traducción de los textos, sino que también se han modificado, por motivos, o bien de interés propio de la campaña o por motivos de adaptabilidad a la cultura meta.

Otro ejemplo de la adaptabilidad a la cultura meta que señalamos en el análisis de todos los textos es cuando el hijo se refiere a la velocidad a la que van al advertirle su madre que van demasiado rápido, y aquí encontramos una 
diferencia entre los textos: tanto el anuncio italiano, como el español y el francés dicen "cuarenta" y suponemos que se refieren a kilómetros, y sin embargo, en el texto traducido al inglés, dice veinte, y suponemos que son millas, por lo que adaptan la velocidad del coche dentro de la ciudad para cada contexto.

Hemos extraído los ejemplos de los textos anteriormente expuestos:

\begin{tabular}{|l|l|}
\hline En el anuncio italiano: & En el anuncio español: \\
\hline $\begin{array}{l}\text { GIGI E dai mamma! Vado a quaranta } \\
\text { all'ora... }\end{array}$ & $\begin{array}{l}\text { GIGI ¡Madona mamma! Ma si voy a } \\
\text { cuarenta... }\end{array}$ \\
\hline En el anuncio francés: & En el anuncio inglés: \\
\hline $\begin{array}{l}\text { GIGI Madona mamma, je roule } \\
\text { seulement à quarante. }\end{array}$ & GIGI Mamma, I'm only doing tuenti \\
\hline
\end{tabular}

La variación diacrónica, como sucede en el texto en italiano, tampoco existe esta variación en el grupo de anuncios en las demás lenguas, debido a las características de nuestro texto de estudio, no existe ninguna variante debida al transcurso del tiempo, no hay arcaísmos ni referencias a las lenguas antiguas en los idiomas respectivos.

La variación diatópica, es una de las características de los textos que más cuesta traducir, porque ¿cómo hacemos para traducir un dialecto?, como es el caso de nuestro anuncio en italiano, ¿qué soluciones tenemos? ¿Elegimos otro dialecto de la lengua traducida?, o simplemente hacemos una traducción estándar en la que se pierden todos los rasgos lingüísticos y culturales que implica utilizar un dialecto.

En nuestro caso, para los anuncios en español, francés e inglés, han mantenido rasgos italianizantes para que se siga teniendo esa fuerza italiana que trasmite el anuncio en italiano.

El caso del nombre del joven protagonista, es un ejemplo del intento de mantener la naturaleza del origen de los personajes, aunque debemos señalar, que sufre una modificación en todos los anuncios traducidos, en el anuncio italiano la madre se refiere al hijo llamándole con el diminutivo Gigi, y en los anuncios traducidos (español, francés e inglés) se refiere a él con el nombre completo Luigi:

La madre se dirige a Gigi de la siguiente forma: 
ENTRECULTURAS Número 3. ISSN: 1989-5097. Fecha de publicación: 12-01-2011

\begin{tabular}{|l|l|l|}
\hline En el anuncio español & En el anuncio francés & En el anuncio inglés \\
\hline $\begin{array}{l}\text { MAMA ¡Luigi no corras } \\
\text { tanto! }\end{array}$ & $\begin{array}{l}\text { MÈRE Luigi, ne roule } \\
\text { pas si vite! }\end{array}$ & $\begin{array}{l}\text { MOTHER Luigi Where } \\
\text { are you going? }\end{array}$ \\
\hline
\end{tabular}

El muchacho se dirige a la madre utilizando la forma italiana : mamma para los tres casos. Veamos algunos ejemplos extraídos de los textos:

Gigi, se dirige así a su madre:

\begin{tabular}{|l|l|l|}
\hline En el anuncio español & En el anuncio francés & En el anuncio inglés \\
\hline GIGI Sí, mamma sí. & GIGI Si, si mamma. & $\begin{array}{l}\text { GIGI Mamma, I'm } \\
\text { only doing tuenti. }\end{array}$ \\
\hline
\end{tabular}

También han empleado palabras en italiano, palabras que son muy características de la lengua italiana, que gracias a su gran difusión se conocen en todo el mundo y es bien sabido que la procedencia es italiana. Aquí plasmamos algunos ejemplos extraídos de los textos:

\begin{tabular}{|c|c|c|}
\hline En el anuncio español: & En el anuncio francés: & En el anuncio inglés: \\
\hline $\begin{array}{l}\text { MAMA Mamma mia, } \\
\text { ¿es que tienes que volar } \\
\text { de esta forma? } \\
\text { GIGI ¡Madona } \\
\text { mamma! Ma si voy a } \\
\text { cuarenta... } \\
\text { GIGI Si, mamma, jun } \\
\text { Red Bull que revitaliza } \\
\text { cuerpo y mente y te da } \\
\text { alas!, Ciao mamma! } \\
\text { MAMA ¡Piensa en tu } \\
\text { mamma! }\end{array}$ & $\begin{array}{l}\text { MÈRE Attention! } \\
\text { Freine, freine, attention, } \\
\text { mamma mia! Mais } \\
\text { pourquoi tu conduis si } \\
\text { vite? } \\
\text { GIGI Estimule le } \\
\text { corps et l'esprit et me } \\
\text { donne des ailes. Ciao } \\
\text { mamma! }\end{array}$ & $\begin{array}{l}\text { GIGI I'm drinking red } \\
\text { bull, it stimulates body } \\
\text { and mind and it gives } \\
\text { you wings. Ciao } \\
\text { Mamma. } \\
\text { MOTHER Think of } \\
\text { your poor mamma }\end{array}$ \\
\hline
\end{tabular}

Como pudimos observar en el anuncio italiano, la madre hace una exclamación a San Genaro, patrón de la ciudad de Nápoles, cuya mención en este texto, hace que aparezca como marca importante para indicar cuál es su origen.

¿Qué sucede en los demás anuncios? Para el resto de los anuncios, esta marca desaparece totalmente, no hacen referencia al santo napolitano, y como 
solución, han preferido no hacer ningún tipo de alusión, se ha optado por neutralizar totalmente la traducción:

\begin{tabular}{|l|l|}
\hline En el anuncio italiano: & En el anuncio español: \\
\hline $\begin{array}{l}\text { MAMMA Ma che quaranta l'ora! } \\
\text { Stiamo volando. Ah! San Gennaro, e } \\
\text { mo' bevi pure mentre guidi, eh? }\end{array}$ & $\begin{array}{l}\text { MAMA ¡El semáforo! iEstá rojo! } \\
\text { iAh! ahora te pones a beber } \\
\text { conduciendo? }\end{array}$ \\
\hline $\begin{array}{l}\text { En el anuncio francés: } \\
\begin{array}{l}\text { MÈRE Mais c'est rouge! Ah! } \\
\text { Et maintenant tu bois aussi en } \\
\text { conduisant! }\end{array}\end{array}$ & $\begin{array}{l}\text { En el anuncio inglés: } \\
\text { Ah! And now you're drinking and } \\
\text { driving! What are you thinking? }\end{array}$ \\
\hline
\end{tabular}

Veamos que ha sucedido con las palabras de origen napolitano que señalamos en el estudio de la traducción de la variación lingüística del texto italiano, en los textos traducidos (español, francés e inglés):

\begin{tabular}{|c|c|}
\hline & En el anuncio español: \\
\hline $\begin{array}{l}\text { MAMMA Ue! Gigí!, ma tu si pazzo! } \\
\text { Non andà così veloce. } \\
\text { GIGI Si... } \\
\text { MAMMA A cintura, te l'hai messa la } \\
\text { cintura... } \\
\text { (...) } \\
\text { MAMMA Va chiano... Frena! Frena! } \\
\text { State accourtu, accussì decolliamo, } \\
\text { bellemammà! } \\
\text { (...) } \\
\text { MAMMA Gigí! A do te ne vai vulando } \\
\text { accussi! Chiama quando arrivi figlio } \\
\text { mio! Va piano, nun fa tard, } \\
\text { m’arraccumandu, pensa a mamma toia } \\
\text { che ti voglie tanto beeeene! }\end{array}$ & $\begin{array}{l}\text { MAMA ¡Luigi no corras tanto! } \\
\text { GIGI Vale... } \\
\text { MAMA ¿Te has puesto el cinturón? } \\
\text { (...) } \\
\text { MAMA ¡Cuidado! ¡frena! ¡Qué } \\
\text { miedo! } \\
\text { Mamma mia, ¿es que tienes que volar } \\
\text { de esta forma? } \\
(\ldots) \\
\text { MAMA ¡Alas! ¡Luigi! ¿Dónde vas? } \\
\text { ¡Llámame cuando hayas llegado! ¡No } \\
\text { vuelvas demasiado tarde a casa! } \\
\text { ¡Piensa en tu mamma! ¡No vueles tan } \\
\text { rápido! }\end{array}$ \\
\hline & \\
\hline $\begin{array}{l}\text { MÈRE Luigi, ne roule pas si vite! } \\
\text { GIGI Si... } \\
\text { MÈRE Tu as mis la ceinture? } \\
(\ldots)\end{array}$ & $\begin{array}{l}\text { MOTHER Atenzione! Brake Brake } \\
\text { Watch out! Mamma mia... Why are } \\
\text { you driving like this? } \\
\text { (...) }\end{array}$ \\
\hline
\end{tabular}




\begin{tabular}{|l|l|}
\hline $\begin{array}{l}\text { MÈRE Attention! Freine, freine, } \\
\text { attention, mamma mia! Mais pourquoi } \\
\text { tu conduis si vite? }\end{array}$ & $\begin{array}{l}\text { MOTHER The lights! No run the } \\
\text { red! Ah! And now you're drinking } \\
\text { and driving! What are you thinking? } \\
(. . .)\end{array}$ \\
$\begin{array}{l}\text { MÈRE Des ailes. Luigi mais où voles- } \\
\text { tu comme ça? Appele-moi quand tu es } \\
\text { arrivé, ne rentre pas trop tard, pense à } \\
\text { ta maman... }\end{array}$ & $\begin{array}{l}\text { Mou going? Call me to let me know } \\
\text { you arrived safely. Don't stay out too } \\
\text { late! Think of your poor mamma! }\end{array}$ \\
\hline
\end{tabular}

Hemos señalado las soluciones que han dado para la traducción de las palabras empleadas en dialecto napolitano en cada texto traducido (español, francés e inglés) y a parte de los matices nuevos y el texto nuevo que le han añadido en las traducciones, como cuando dice al final del texto: $i N o$ vueles tan rápido! en el anuncio español, o Ne vole pas si vite! en el anuncio francés, o Think of your poor mamma! para la traducción inglesa, y que en el anuncio italiano no existe, por lo demás, las soluciones para la traducción del dialecto napolitano han sido neutralizar la traducción; no aparece ninguna señal de que la lengua en la que se expresa la madre es en dialecto.

Exceptuando las palabras italianas que mantienen en italiano, y el fuerte acento italiano que tienen los dos personajes, los ejemplos que hemos señalado para analizar, se pierden, el trasvase cultural y lingüístico desaparece en este caso; podríamos decir que la solución es que cuando aparece alguna expresión o palabra en dialecto napolitano, la solución es o bien, neutralizarla, o bien variar la traducción y añadir o eliminar texto, porque de todas formas, el público no va a percatarse del cambio.

La variación diastrática se encuentra en los tres modelos de anuncio, el lenguaje que utilizan, hace que pertenezcan a dos grupos sociales diferentes: diferenciados por la edad y el sexo la madre entra un grupo social y Gigi, responde a otro grupo social. La edad es un elemento que determina la elección lingüística del hablante. Aunque a menudo sea imposible determinar cuales, en cuanto a los jóvenes en Italia no hablan un italiano muy diferente de los mayores, la edad puede determinar el uso de ciertas variedades lingüísticas. Muchas formas lingüísticas están de igual manera disponibles a todos los hablantes, aunque a menudo son elegidas y usadas sobre todo por los jóvenes, a diferencia de otras que lo son para los mayores. (Caprara, 2007).

Aquí plasmamos algunos ejemplos extraídos de los textos: 
ENTRECULTURAS Número 3. ISSN: 1989-5097. Fecha de publicación: 12-01-2011

\begin{tabular}{|l|l|l|}
\hline En el anuncio español: & En el anuncio francés: & En el anuncio inglés: \\
\hline $\begin{array}{l}\text { MAMA ¡Alas! ¡Luigi! } \\
\text { ¿Dónde vas? ¡Llámame } \\
\text { cuando hayas llegado! } \\
\text { ¡No vuelvas demasiado } \\
\text { tarde a casa! ¡Piensa en } \\
\text { tu mamma! ¡No vueles } \\
\text { tan rápido! }\end{array}$ & $\begin{array}{l}\text { pourquoi tu conduis si } \\
\text { vite? }\end{array}$ & $\begin{array}{l}\text { MOTHER Attention! } \\
\text { Brake Brake! Watch out! }\end{array}$ \\
\hline GIGI Vale... & $\begin{array}{l}\text { GIGI Madona mamma, attention, } \\
\text { je roule seulement a } \\
\text { quarante. }\end{array}$ & $\begin{array}{l}\text { GIGI Mamma, I'm only } \\
\text { doing tuenti }\end{array}$ \\
\hline
\end{tabular}

Ambos pertenecen al grupo social de personas extranjeras, en este caso italianas, que viven en otro país, al mezclar en la conversación ambos idiomas.

Esta característica también se ve claramente cuando visionamos y escuchamos el anuncio; los personajes tienen un acento italiano muy exagerado en los tres idiomas en los que estamos trabajando. En el caso del anuncio inglés, es un acento muy fuerte, y cabe señalar las dificultades que hemos tenido para realizar la trascripción de la parte inicial, cuando interviene la madre en el primer párrafo.

La variación diamésica, como ya hemos visto en el estudio del anuncio en italiano, tiene como característica la distinción entre el medio utilizado, y al igual que el italiano, el resto de anuncios también se caracterizan por utilizar un tipo de lenguaje con características del lenguaje oral, más fluido y menos preocupado que el lenguaje escrito.

Aquí mostramos algunos ejemplos extraídos de los textos:

\begin{tabular}{|l|l|l|}
\hline En el anuncio español: & En el anuncio francés: & En el anuncio inglés: \\
\hline $\begin{array}{l}\text { MAMA ¡Alas! ¡Luigi! } \\
\text { ¿Dónde vas? }\end{array}$ & $\begin{array}{l}\text { MÈRE Mais c'est rouge! } \\
\text { Ah! }\end{array}$ & $\begin{array}{l}\text { MOTHER Attention! } \\
\text { Brake Brake! Watch out! }\end{array}$ \\
\hline GIGI Vale... & GIGI Si... & $\begin{array}{l}\text { GIGI I'm only doing } \\
\text { tuenti }\end{array}$ \\
\hline
\end{tabular}

La variación diafásica, en los anuncios que aquí estudiamos, sigue las mismas pautas que el anuncio en italiano: ha sido pensada para que tengan un lenguaje adaptado a una situación comunicativa de ámbito cotidiano, sin ninguna especialización, con un vocabulario muy normal.

El campo no es en ninguno de los casos especializado, el tono es muy familiar, con muy poca distancia entre ellos y el modo o canal de comunicación es el oral, aunque tenemos que tener en cuenta, como en el estudio que hemos hecho anteriormente sobre el anuncio italiano, que se trata de una campaña 
publicitaria, y para cada anuncio, ha habido un estudio previo, y aunque se han pensado para ser anuncios, ha habido una previa escritura de los textos, que han sido adaptados para ser interpretados por actores de doblaje. Debemos señalar que en todos los textos, italiano, español, francés e inglés, existe la misma frase de la campaña publicitaria y cuya traducción no ha sufrido ninguna variedad, es una traducción estándar para que el mensaje se trasmita correctamente y no tenga interferencias. En el caso italiano, dicha frase está expresada en italiano estándar. Aquí señalamos los ejemplos obtenidos de los textos para cada una de las frases del slogan del anuncio:

\begin{tabular}{|c|c|c|}
\hline En el anuncio español: & En el anuncio francés: & En el anuncio inglés: \\
\hline $\begin{array}{l}\text { MAMA ¡Alas! } \\
\text { ¡Luigi! ¿Dónde vas? } \\
\text { ¡Llámame cuando hayas } \\
\text { llegado! ¡No vuelvas } \\
\text { demasiado tarde a casa! } \\
\text { ¡Piensa en tu mamma! } \\
\text { ¡No vueles tan rápido! }\end{array}$ & $\begin{array}{l}\text { MÈRE Des } \\
\text { ailes. Luigi mais où } \\
\text { voles-tu comme ça? } \\
\text { Appele-moi quand tu es } \\
\text { arrivé, ne rentre pas } \\
\text { trop tard, pense à ta } \\
\text { maman... Ne vole pas } \\
\text { si vite! }\end{array}$ & $\begin{array}{l}\text { MOTHER } \\
\text { Luigi Where are you } \\
\text { going? Call me to let me } \\
\text { know you arrived safely. } \\
\text { Don't stay out too late! } \\
\text { Think of your poor } \\
\text { mamma! }\end{array}$ \\
\hline $\begin{array}{l}\text { GIGI } \\
\text { mamma, iun Red Bull } \\
\text { que revitaliza cuerpo y } \\
\text { mente y te da alas!, Ciao } \\
\text { mamma! }\end{array}$ & $\begin{array}{l}\text { GIGI Si } \\
\text { mamma, c'est du Red } \\
\text { Bull! Estimule le corps } \\
\text { et l'esprit et me donne } \\
\text { des ailes. Ciao mamma! }\end{array}$ & $\begin{array}{l}\text { GIGI That's } \\
\text { right, I'm drinking red } \\
\text { bull, it stimulates body } \\
\text { and mind and it gives } \\
\text { you wings. } \\
\text { Mamma. Ciao } \\
\text { Mau }\end{array}$ \\
\hline
\end{tabular}

\section{CONCLUSIONES}

Tras haber realizado el estudio de la traducción de la variación lingǘstica del anuncio en italiano y haber hecho la comparativa con los anuncios en español, francés e inglés, podemos obtener como conclusiones varias ideas:

Teniendo en cuenta que se trata de una campaña publicitaria a nivel europeo, han sabido mantener el fondo y la forma de la idea del anuncio, a excepción del anuncio traducido al inglés, que han decidido variar un poco el diálogo en el que participa la madre al inicio de su intervención. Indicamos que han adaptado los diálogos a las culturas meta, sobre todo para el anuncio en inglés. 
Cuando nos referimos al fondo, estamos pensando no sólo a la campaña publicitaria en sí misma y a las ventas del producto, que de eso ya se han encargado los publicistas, sino al fondo del propio anuncio, han realizado un producto con una intención cómica y esa intención tienen que llegar al receptor, en este caso, a todo el público. Para conseguir este objetivo, han sabido mantener la esencia en todos los modelos, a través del fuerte acento italiano de la madre y las expresiones italianas utilizadas por ambos en todos los anuncios traducidos, aunque debemos apuntar que el anuncio en italiano consigue de manera más eficaz lo que pretenden, ya que partiendo de la posibilidad que el juego y la combinación del idioma italiano con el dialecto napolitano, les permite intensificar al máximo toda la situación cómica, casi teatral del anuncio.

Cuando nos referimos a la forma, hablamos de la forma en sí de los cuatro anuncios, han hecho una traducción del anuncio original en vez de crear cuatro campañas diferentes; por lo tanto, han tenido que tener en cuenta muchos factores lingüísticos para que los cuatro anuncios trasmitan lo mismo para cada cultura meta, culturas diferentes, y en cada caso han tenido que adaptarse a cada una de ellas, manteniendo la idea principal del anuncio: que se mantenga la característica principal de los personajes, que son italianos.

En los anuncios no italianos, se ha conseguido mantener esta característica, gracias a que el medio de transmisión del mensaje final es audiovisual, y permite jugar con la acentuación en cada caso, y lo que obtenemos es una clara identificación de los personajes con el habla italiana.

Esta característica no es necesaria en el anuncio italiano, ya que es obvio que el anuncio va a ser en italiano, por eso, es un buen recurso el uso del dialecto napolitano, que hace que el anuncio en sí logre un resultado excepcional.

Poder reflejar las variaciones lingǘsticas del mismo anuncio de base en anuncios de diferentes idiomas y de diferentes culturas, no es tarea sencilla, pero si se logra, como es el caso de esta campaña publicitaria, se obtiene un resultado de gran calidad, y aunque no sea el camino más fácil, si es el mejor, por lo que, los traductores de este tipo de trabajos, deberíamos tener más en cuenta la traducción de la variación lingǘstica, para que la calidad de los trabajos sea mucho mejor aún.

Después de haber realizado este estudio, podemos dar respuesta a las dudas que nos surgieron al inicio del mismo y que fueron parte de las motivaciones para realizarlo; nos hemos preguntado el por qué creemos que es necesario estudiar la traducción de la variación lingüística en los medios audiovisuales, y la respuesta ahora nos resulta evidente, la variación lingüística y todos sus tipos, son los elementos que nos dan todas las informaciones que no nos dan las palabras por sí solas, y un texto audiovisual, como el resto de textos, necesita, para poder trasmitir toda su esencia, de la variación lingüística 
y que ésta se traduzca para poder realizar el trasvase de la cultura de origen a la cultura meta, si no en su totalidad, por lo menos parcialmente, creemos que es importante.

También nos preguntábamos el por qué de haber elegido este anuncio publicitario y esta pregunta tiene múltiples respuestas, primero por su forma, ya que es un formato atractivo, nos pareció simpático y se ajustaba a nuestras necesidades para hacer esta investigación.

Segundo, porque obtuvimos todos los anuncios (en los cuatro idiomas que queríamos trabajar) de una forma muy accesible, gracias a Ana Fernández de la agencia Kastner Network, que nos los envió muy amablemente y pudimos trabajar con ellos en excelentes condiciones.

Y tercero, elegimos este anuncio, por la gran riqueza lingüística que nos proporcionaron para realizar el estudio de la variación lingüística y su traducción: el anuncio italiano que combina la lengua italiana estándar con el dialecto napolitano; y los anuncios traducidos, que mantienen algunos rasgos italianizantes que queríamos analizar para comprobar si realmente se mantenía la variedad lingüística en el trasvase de un idioma a otro.

Nuestra siguiente pregunta era si se lograba el trasvase de la variación lingüística en este medio audiovisual, y así como nos pareció en un principio, es una pregunta muy amplia, que realmente no se puede contestar con sólo el estudio de un solo ejemplo, pero muy humildemente, podemos analizar los resultados de nuestra investigación, y pensamos que se mantienen algunos rasgos, de la naturaleza de los personajes del anuncio, unos rasgos muy generales como el acento, que es muy fuerte para el caso de la madre, y algunas palabras italianas que siguen apareciendo en los anuncios traducidos, como ya hemos indicado anteriormente en el artículo.

Pero queremos señalar que, la variedad diatópica, el origen napolitano de los hablantes en el anuncio italiano, se pierde en su totalidad en los anuncios traducidos. Por lo que el trasvase cultural no se ha logrado plenamente, sólo se ha trasmitido un origen italiano, pero todas las marcas dialectales se han perdido.

La última pregunta que nos hacíamos era cuáles posibles soluciones existen para la traducción del dialecto, y como respuesta, al igual que la anterior, sólo podemos basarnos en nuestra investigación y obtenemos muy pocos resultados, porque como ya hemos apuntado en el punto precedente, el dialecto napolitano se pierde en su totalidad. Sólo existe la marca del acento, pero no la procedencia exacta de los personajes.

Pregunta que decidimos dejar abierta para posibles futuras investigaciones dentro el ámbito de la variación lingüística y su traducción en los medios audiovisuales. 


\section{BIBLIOGRAFÍA}

Agost, Rosa (1998): "La importància de la variació lingüística en la traducción". Quaderns. Revista de Traducció, n. ${ }^{\circ}$ 2, 83-95.

- (1999): Traducción y doblaje: palabras, voces e imágenes. Barcelona, Ariel.

Baccolini, Rafaella; Rosa María Bollettieri Bosinelli y Laura Gavioli (1994): Il doppiaggio. Trasposizioni linguistiche e culturali. Forlí, Scuola Superiore Traduttori e Interpreti.

BAKER, Mona (1992): In Other Words: A Coursebook on Translation. Londres, Routledge. 1992.

Blomfield, Leonard (1933): Language. Nueva Cork, Holt, Rinehart and Winston.

CATFORD, John (1965): A Linguistic Theory of Translation. Londres, Oxford University Press, 1965.

CAPrara, Giovanni (2007): Variación lingüistica y traducción: Andrea Camilleri en castellano. Tesis doctoral. Inédito. Málaga: Universidad de Málaga.

Chaume Varela, Frederic (2004): Cine y Traducción. Madrid, Cátedra.

Coseriu, Eugenio (1981): Lecciones de lingüistica general. Madrid, Gredos.

Crystal, David y Derek DAvy (1969): Investigating English style. Londres, Longman.

FIRTH, John (1935): The tecnique of semantics.

Hallyday, M.A.K; Angust McIntosh Angust y Meter STREVENs (1966): The linguistic sciences and language teaching. Londres, Longman.

HATIM, Basil e Ian MASON (1997): The translator as communicator. Londres/Nueva York, Routledge.

- (1990): Discourse and Translator. Londres, Longman.

HiCKey, Leo (1987): Curso de Pragmaestilística. Madrid, Coloquio.

Hurtado Albir, Amparo (2001): Traducción y traductología. Introducción a la traductología. Madrid, Cátedra.

LABOV, William (1966): The social stratification of English in New Cork City. Washington D.C., Center of Applied Linguistics.

LÓPEZ Morales, H.: "La sociolingüística actual" en Estudios sobre variación lingüística. Alcalá de Henares, Servicio de Publicaciones de la Universidad de Alcalá de Henares.

Mayoral Asensio, Roberto (1990): "Comentario a algunas de las variedades de lengua". Sendebar, n. ${ }^{\circ}$ 1, 35-46.

- (1990): La traducción de la variación lingüística. Monográfico de la revista Hermeneus, n. ${ }^{\circ} 1$. Soria.

- (1989): "Problemas de traducción de los sistemas de referencia de segunda y tercera persona". Sevilla, AESLA. 
- (1987): "El texto como unidad en la traducción del tabú lingüístico". Lenguaje y educación. Córdoba, AESLA.

Mayoral, R.; D. Kelly Y N. Gallardo (1986): "Concepto de 'traducción subordinada' (cómic, cine, canción, publicidad). Perspectivas no lingüísticas de la traducción (I)" en F. Fernández (ed.).

MichaEL, Gregory y Susanne CARROLL (1978): Language and Situation: Language Varieties and Their Social Contexts. Londres, Routledge and Kegan Paul.

MounIN, Georges (1977): Los problemas teóricos de la traducción. Madrid: Gredos.

NIDA, Eugene: "Varieties of language" en Language Structure and Translation. Standford: Standford University Press, 174-183.

- (1947): Languages and Dialects into which Translations Should Be Made.

- (1982): Reestructuring.

RABADÁN (1991): Equivalencia y traducción: problemática de la equivalencia translémica inglés-español. León, Universidad de León.

SAussure, Ferdinand de (1916): Cours de linguistique genéral. París: Payot.

VALDÉS, Cristina (1998): "Estrategias traductológicas de los textos publicitarios televisivos» en Orero. P.

Vergara, Gaetano (2001): “Teng'Suonno. Analogías y semejanzas entre el español y el napolitano". Extracto de las actas recogidas de una ponencia para la Asociación cultural hispano-italiana La Tertulia. 20 de Mayo de 2001. 